\title{
Spontaneous EEG oscillations reveal periodic sampling of visual attention
}

\author{
Niko A. Busch ${ }^{\mathrm{a}, \mathrm{b}, 1}$ and Rufin VanRullen ${ }^{\mathrm{c}, \mathrm{d}}$

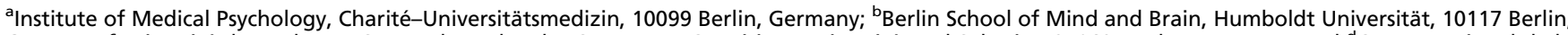 \\ Germany; ' Université de Toulouse, Centre de Recherche Cerveau et Cognition, Université Paul Sabatier, 31062 Toulouse, France; and ${ }^{\mathrm{d} C e n t r e ~ N a t i o n a l ~ d e ~ l a ~}$
} Recherche Scientifique, Unité Mixte de Recherche 5549, Faculté de Médecine de Toulouse Rangueil, 31062 Toulouse, France

Edited by Dale Purves, Duke University Medical Center, Durham, NC, and approved August 10, 2010 (received for review April 9, 2010)

\begin{abstract}
An important effect of sustained attention is the facilitation of perception. Although the term "sustained" suggests that this beneficial effect endures continuously as long as something is attended, we present electrophysiological evidence that perception at attended locations is actually modulated periodically. Subjects detected brief light flashes that were presented peripherally at locations that were either attended or unattended. We analyzed the correlation between detection performance for attended and unattended stimuli and the phase of ongoing EEG oscillations, which relate to subsecond fluctuations of neuronal excitability. Although on average, detection performance was improved by attention-indicated by reduced detection thresholds at attended locations-we found that detection performance for attended stimuli actually fluctuated over time along with the phase of spontaneous oscillations in the $\theta(\approx 7 \mathrm{~Hz})$ frequency band just before stimulus onset. This fluctuation was absent for unattended stimuli. This pattern of results suggests that "sustained" attention in fact exerts its facilitative effect on perception in a periodic fashion.
\end{abstract}

O ur senses are constantly confronted with a flow of information that exceeds the limits of our sensory systems. One mechanism that limits this input to a manageable amount is selective attention - the ability to enhance processing of a particular object or location. The investigation of attention has been central to our understanding of cognition. Here we present evidence for a largely unexplored feature of selective attention: its periodic rather than continuous operation.

The most prominent effect of attention is the facilitation of perception-selected information is processed more efficiently $(1,2)$ or faster $(3)$. One example of this facilitation is enhanced contrast sensitivity at attended locations $(4,5)$. Two types of selective attention are usually distinguished: transient automatic attention and sustained voluntary attention $(6,7)$. Transient attention is captured automatically by sudden salient events, whereas sustained attention can be controlled at will. Although the term "sustained" suggests that the facilitative effect of attention endures continuously as long as attention is deployed at a particular location, it is conceivable that attention operates in a periodic rather than a continuous fashion $(8,9)$. This periodicity of attention is implicit in many visual search tasks in which attention is assumed to switch successively between the different display elements (10-12). On the basis of modeling of human psychometric functions in a signal detection task, VanRullen et al. (8) recently proposed that attention could sample information at a rate of approximately seven items per second-no matter whether attention was focused on multiple targets or on just a single item. A similar attention-driven periodic sampling mechanism (albeit at a different frequency of $\approx 13 \mathrm{~Hz}$ ) has been suggested to account for illusory motion reversals in the continuous wagon-wheel illusion $(9,13,14)$.

Periodic processes are not only found in psychophysical data; they are very prominent in electrophysiological recordings. Here, oscillations correspond to periodic fluctuations of the local electrical field and the intrinsic excitability of neuronal populations (15-21). Of course, these electrical neural oscillations could provide the physiological basis of periodic perceptual and attentional sampling phenomena (22). Recently, two studies have demonstrated that perceptual performance fluctuates between favorable and less-favorable periods along with the phase of $7-10 \mathrm{~Hz}$ oscillations of the human EEG $(23,24)$.

In the present study we investigated how this connection between ongoing EEG oscillations and perception is modulated by selective attention to reveal possible fluctuations of "sustained" attention. Human observers detected luminance targets that were presented at threshold either at attended or unattended locations. In keeping with the idea that "sustained" attention in fact operates in a periodic fashion, we found that detection performance was correlated with the phase of ongoing EEG oscillations around $7 \mathrm{~Hz}$, but only for stimuli presented at attended locations.

\section{Results}

Human observers detected luminance targets that were presented either to the left or to the right of a central fixation cross (Fig. 1). At the start of each run of trials, a symbolic cue was presented at the center of the screen, which instructed observers to focus their attention to the left or to the right. Targets were then presented either at the cued location (valid; attended) or at the uncued location (invalid; unattended) and had to be detected regardless of where they appeared. The luminance of the stimuli was adjusted by running staircase procedures, such that observers detected only half of the stimuli (hits) and entirely missed the other half (misses).

Behavioral Results. Attention was effectively allocated on the cued location, as assessed by the lower detection threshold for cued vs. uncued stimuli $\left[F_{(1,12)}=18.77 ; P<0.0001\right.$; Fig. $\left.1 A\right]$. There was also a tendency for thresholds to be lower in the left hemifield $\left[F_{(1,12)}=9.68 ; P=0.009 ;\right.$ no interaction with attention $]$. The staircase procedures were successful at keeping detection performance fixed at $50 \%$ in all experimental conditions (attended and unattended) and across hemifields (left and right). The overall false-alarm rate was $1.8 \%$.

Electrophysiological Results. Event-related potential (ERP) amplitudes were strongly affected by perception (Fig. $2 A$ ) - for hits, two main ERP components were found: a negative component peaking at $240 \mathrm{~ms}$ with a maximum at posterior electrodes and a positive component peaking at $460 \mathrm{~ms}$ with a posterior-parietal topography. These ERPs were virtually absent when the target was shown but not perceived. Furthermore, amplitudes of these two ERP components were larger for attended than for un-

Author contributions: N.A.B. and R.V. designed research; N.A.B. performed research N.A.B. analyzed data; and N.A.B. and R.V. wrote the paper.

The authors declare no conflict of interest.

This article is a PNAS Direct Submission.

${ }^{1}$ To whom correspondence should be addressed. E-mail: niko.busch@hu-berlin.de.

This article contains supporting information online at www.pnas.org/lookup/suppl/doi:10 1073/pnas.1004801107/-/DCSupplemental. 

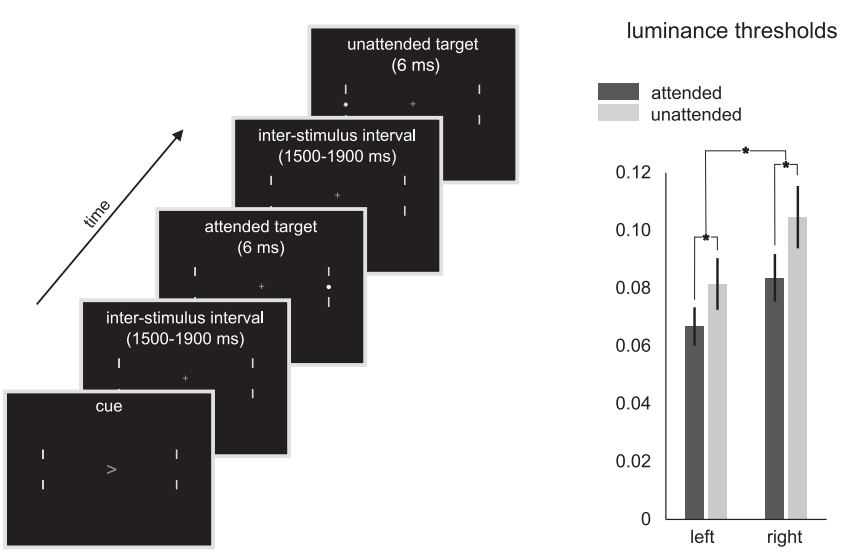

Fig. 1. (Left) illustration of the experimental paradigm. Each run of trials consisted of a symbolic cue followed by four targets at the cued location (attended), four targets at the uncued location (unattended), and one catch trial without a target in randomized order. Note that size and luminance of the targets are magnified in this illustration. Right: (Right) $50 \%$ detection thresholds as estimated by the staircase procedures as a function of hemifield (left/right) and attention (attended/unattended). Thresholds were lower for attended stimuli and for stimuli presented in the left hemifield. Significant effects are marked with asterisks; error bars represent SEM.

attended detected stimuli. These effects were confirmed for both components by significant ANOVA effects for perception $\left[F_{(1,12)}=\right.$ 85.17; $P<0.0001$; and $F_{(1,12)}=87.84 ; P<0.0001$; early and late component, respectively] and for attention $\left[F_{(1,12)}=10.74 ; P=\right.$ 0.007 ; and $\left.F_{(1,12)}=5.29 ; P=0.040\right]$ and by significant perception $\times$ attention interactions $\left[F_{(1,12)}=7.84 ; P=0.016\right.$; and $F_{(1,12)}=9.26$; $P=0.010]$.

We exploited this tight connection between amplitude of the EEG response and perception by using single-trial EEG responses as a
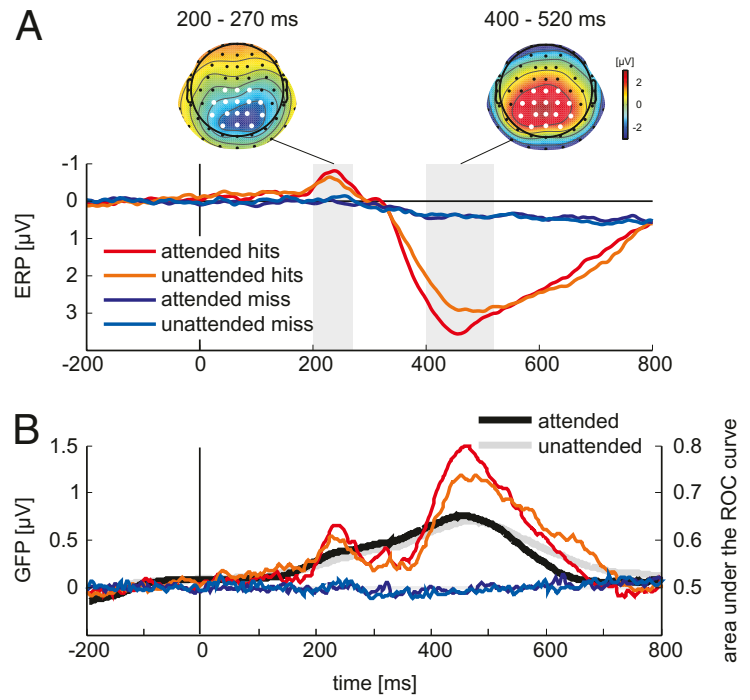

Fig. 2. (A) ERPs (grand average over 13 subjects). Time courses show data averaged across electrodes in a posterior-parietal region of interest (indicated by white markers in the topographies). Shaded time windows indicate the time ranges used for statistical analysis of the ERP. Topographies show ERP distributions in these time ranges averaged across attended and unattended hits. ( $B$ ) GFP (colored lines) and area under the ROC curve (black/ gray line). The time course of this area measure for each subject provides information about when the GFP contains the most information about perception (hits vs. misses). proxy for perception. If perception (and thus amplitude of the EEG response) fluctuates along with ongoing EEG oscillations, the phase of the EEG oscillation at stimulus onset should be correlated with the poststimulus EEG response on a trial-by-trial basis. As a measure for the EEG response, we used global field power (GFP), which is a global measure of the electrophysiological response to a stimulus that does not depend on an arbitrary selection of electrodes. Because phase is a circular measure (e.g., $0^{\circ}=360^{\circ}$ ), traditional linear statistics such as the Pearson correlation coefficient cannot be used; circular statistics have to be used instead $(25,26)$. Here we used the circular-to-linear correlation (SI Materials and Methods) between prestimulus phase (circular) and poststimulus GFP (linear). The rationale of the circular-to-linear correlation analysis is illustrated in Fig. S1. For each subject, the GFP was quantified in the time window in which it provided maximal information about perception. This time window was determined by computing a receiver operating characteristic (ROC) analysis (SI Materials and Methods), which yielded a measure of the accuracy by which a subject's perception in the detection task can be predicted from the GFP on a trial-by-trial basis. On average, GFP was most predictive of perception at $432 \mathrm{~ms}$ (SD: $146 \mathrm{~ms}$; Fig. 2B). Correlations between the GFP at this time and prestimulus phase were first computed for each condition (attended left, attended right, unattended left, unattended right) and channel separately and then averaged. Across all channels, this analysis yielded strong correlations between prestimulus phase and poststimulus GFP in a window from 4 to $10 \mathrm{~Hz}$ and $-400 \mathrm{~ms}$ to -100 $\mathrm{ms}$. The effect in this time-frequency window had a fronto-central topography (Fig. $3 A$ ). Next, correlations were considered separately for attended and unattended stimuli at fronto-central channels (Fig. $3 B$ ). Here, the circular-to-linear correlation between prestimulus phase and GFP was considerably stronger for attended than for unattended stimuli. This was confirmed by a two-tailed $t$ test, which compared the magnitude of the circular-to-linear correlation coefficient $\rho$ at these channels averaged across the time-frequency
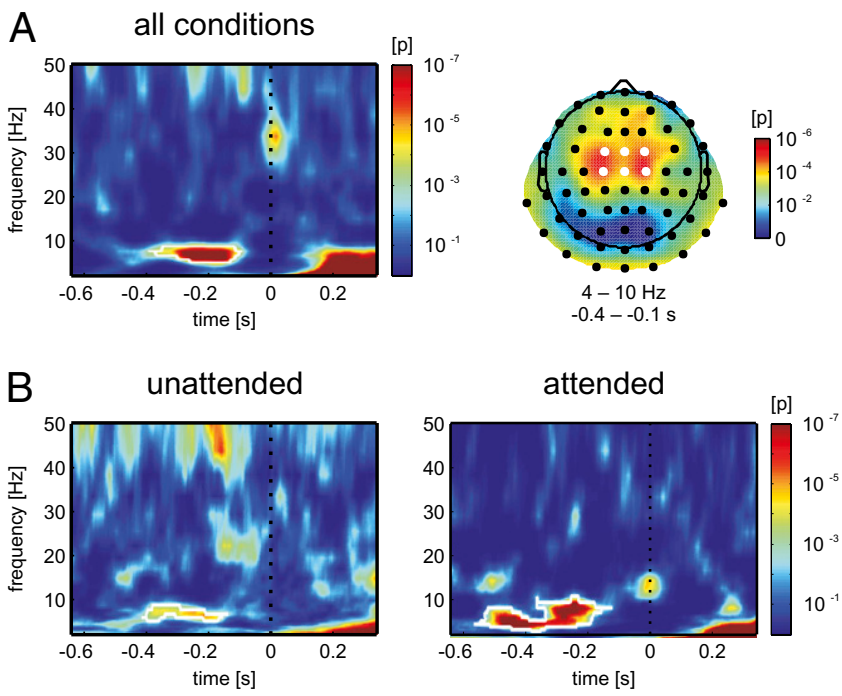

Fig. 3. Circular-linear correlation between EEG phase and poststimulus global field power. $(A)$ Correlation computed across all conditions and channels. The color map represents $P$ values, and the white outline delimits significant effects corresponding to a FDR of $5 \%$. Correlations are most evident around $7 \mathrm{~Hz}$ at $400-100$ ms prestimulus, and at frequencies $<5 \mathrm{~Hz}$ after $100 \mathrm{~ms}$ poststimulus (corresponding to the early portion of the ERP). The topography shows the distribution of the correlation in the time-frequency range from 4 to $10 \mathrm{~Hz}$ and -400 to $-100 \mathrm{~ms}$. White markers indicate channels of the region of interest, used for subsequent analyses. $(B)$ Correlations separately for unattended and attended stimuli computed for the fronto-central region of interest. Stronger and more sustained correlation between prestimulus phase and GFP is found for attended stimuli. 
window from 4 to $10 \mathrm{~Hz}$ and $-400 \mathrm{~ms}$ to $-100 \mathrm{~ms}\left[t_{(24)}=2.63 ; P=\right.$ $0.015]$. A difference was also found when the test was performed separately for each time-frequency point rather than on the average in a time-frequency range (Fig. S2).

The magnitude of the attention effect on prestimulus phase was assessed by pooling single trials according to the phase at the optimal time-frequency point $(-224 \mathrm{~ms}, 7.1 \mathrm{~Hz})$ and computing the average GFP (Fig. $4 A$ ) and detection rate (Fig. $4 B$ ) in each of 11 bins. For each subject, phase angles were shifted such that the phase at which GFP amplitudes were largest was aligned to a phase angle of zero. As a result of this alignment, GFP is necessarily maximal at a phase angle of $0^{\circ}$. Most importantly, GFP amplitudes monotonically decreased to a minimum at the opposite phase angle, confirming that prestimulus phase affected poststimulus neural responses. This effect was confirmed statistically by a significant phase factor: $\left[F_{(9,117)}=7.188 ; P<0.0001\right.$; excluding the $0^{\circ}$ phase bin]. Moreover, this periodic modulation of GFP was stronger for attended than for unattended stimuli. This was confirmed by a phase $\times$ attention interaction: $F_{(9,117)}=4.025 ; P=$ 0.0002 . Post hoc tests revealed that, in fact, prestimulus phase affected GFP only for attended $\left[F_{(9,117)}=5.549 ; P<0.0001\right]$ but not unattended stimuli $\left[F_{(9,117)}=1.583 ; P=0.128\right]$. Comparing the average of the two bins adjacent to the zero bin with the average of the two bins at the opposite phase revealed that for attended stimuli, phase accounted for a difference of $9 \%$ of the GFP. These findings could be confirmed by analyzing plain ERP waveforms, which were largest when stimuli were preceded by the "preferred" phase, smallest for the "opposite" phase, and in between for "intermediate" phases (Fig. S3).

A similar pattern of results was found for perceptual performance (Fig. 4B). Although no main effect of phase was found $(F<1)$, a phase $\times$ attention interaction indicated that perception of attended stimuli was more strongly modulated than unattended stimuli $\left[F_{(9,117)}=2.663 ; P=0.0076\right]$. This was confirmed by post hoc tests that revealed a significant phase effect only for attended stimuli $\left[F_{(9,117)}=3.006 ; P<0.0029\right]$ but not for unattended stimuli $(F<1)$. For attended stimuli, the difference between the average of the two bins adjacent to the zero bin and the average of the two bins at the opposite phase revealed that phase accounted for a difference of $11 \%$ of performance. Because the phase alignment had adjusted
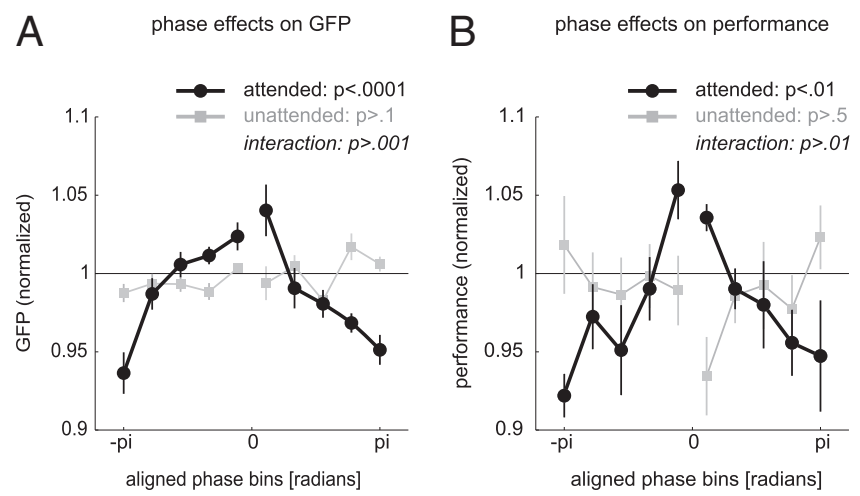

Fig. 4. GFP and perceptual performance as a function of prestimulus phase at $7.1 \mathrm{~Hz}$ and $-224 \mathrm{~ms}$. (A) Relationship between prestimulus phase and GFP for attended and unattended stimuli. Single trials were pooled in 11 bins, centered on the preferred phase bin. Statistical tests compare the 10 nonzero bins, leaving aside the central bin. The curves indicate that GFP amplitudes for attended, but not for unattended stimuli, decrease monotonically the more phases deviate from the preferred phase angle. Error bars represent SEM. $(B)$ Relationship between prestimulus phase and perceptual performance (hit rate) for attended and unattended stimuli. Conventions as in $A$. Detection performance for attended stimuli (but not for unattended stimuli) decreases monotonically the more phases deviate from the preferred phase angle. phase distributions according to the phase yielding largest GFP responses, this result indicates that the same phase is predictive for both the GFP response and for perception.

To analyze phase effects on perception directly, we investigated whether the phase distributions for hits and misses were locked to different phase angles using a Watson-Williams test $(25,26)$. This test showed that hits and misses were indeed associated with different phase angles, but only for attended stimuli (Fig. S4). Moreover, this effect was found in the same time-frequency region as identified by virtue of the phase-GFP correlation (see above). Thus, the direct comparison of phase distributions for hits and misses and the correlation of prestimulus phase and GFP converged at the same principal finding: perception is facilitated by attention, but performance (hit rate) is not continuously facilitated while attention is deployed at a location. Rather, the hit rate for attended stimuli fluctuates periodically along with oscillations of $\approx 7 \mathrm{~Hz}$.

To determine whether phase effects were linked to concurrent changes in oscillatory power, we compared the prestimulus power for hits vs. misses (Fig. S5A) and for attended vs. unattended stimuli (Fig. S5B). Whereas effects of prestimulus phase were most pronounced in the frequency band from 7 to $10 \mathrm{~Hz}$ at frontocentral channels, effects of prestimulus power were strongest in the classical $\alpha$ band from 8 to $15 \mathrm{~Hz}$. Moreover, the scalp distribution of these power effects had a posterior topography. Therefore, phase and power effects on perception seem to be largely independent. Our analyses also confirmed well-known properties of $\alpha$ oscillatory power (27-31): prestimulus $\alpha$ power was significantly stronger preceding misses compared with hits (Fig. S5A) throughout the prestimulus window $\left[t_{(24)}=-3.4806 ; P=0.002\right]$; furthermore, the instruction to focus attention at a particular location significantly increased prestimulus $\alpha$ power at posterior channels ipsilateral to the to be attended site $\left[t_{(24)}=-4.6174 ; P=\right.$ 0.0001 ; Fig. S5B].

\section{Discussion}

We investigated how attention influences the sampling of visual information to test whether the facilitative effects of attention on perception are continuous or rather periodic. Subjects detected threshold stimuli, which were presented at attended or unattended locations. Periodic sampling was quantified as the correlation between the phase of prestimulus EEG oscillations and the detection of these stimuli. Electrophysiological oscillations reflect periodic variations of membrane potentials in neuronal ensembles, which are associated with variations of neural excitability $(15,16,20,32)$. Thus, the momentary phase of ongoing oscillations at the time a stimulus is presented can influence the strength of the neural response to the stimulus (19, 33-37) and the speed of manual reaction times (19, 38, 39). Most importantly, phase can also influence whether a stimulus is perceived at all $(23,24)$, indicating that the visual detection threshold is not constant over time but fluctuates along with the phase of spontaneous EEG oscillations. As a result, such excitability cycles may instantiate a discrete processing mode, framing visual perception into discrete epochs or "perceptual moments" $(13,22,40,41,42)$. In the present study, we confirmed previous reports of correlations between prestimulus phase and perception $(23,24)$ but found evidence that this periodic sampling exists only for attended, but not for unattended, stimuli. This suggests that the beneficial effect of attention (i.e., improved detection thresholds) is not sustained but actually periodic.

The experimental paradigm used in this study required participants to direct attention to either of two locations according to an endogenous cue, and to keep attention deployed at that location while a stream of stimuli was presented at either the attended or an unattended location. Given that the cue was in fact not predictive of the occurrence of stimuli, it is important to find independent evidence that subjects actually deployed their 
attention at the indicated location. We found three effects that provide convincing evidence that the cue was successful in directing subjects' attention to the to-be-attended locations. First, contrast thresholds were lower for stimuli presented at attended locations. This effect is in line with studies demonstrating that attention increases contrast sensitivity $(4,5,43)$. Second, ERP amplitudes were larger in response to stimuli presented at attended locations. This effect of cue validity has been reported repeatedly as a correlate of spatial selective attention $(44,45)$. Third, even before stimulus onset, we found that oscillatory EEG activity in the $\alpha$ range was stronger at electrodes ipsilateral to the to-be-attended location. Similar effects have been reported in cuing studies $(30,31)$. Enhanced $\alpha$ activity is thought to inhibit processing in cortical regions that are not (or less) functionally relevant in a given task (46). Together, these results confirm that participants properly focused attention at the cued locations. Nevertheless, future studies should be conducted to confirm and extend our results. In these studies, the effect of the attentional manipulation could be maximized by making the cue informative (i.e., by presenting more stimuli at the cued location than at the uncued location). This would give subjects more incentive to focus their attention at the cued location. Furthermore, different response categories could be used for left and right targets, and a larger number of blank trials could be presented, allowing for a more precise estimation of the false-alarm rate. However, as long as stimuli at uncued locations are to be reported and thus are task relevant, it will be difficult to ensure that these stimuli are really completely unattended. The present findings might delineate a way to circumvent this problem. We found that measureable EEG responses are obtained only for detected but not for missed stimuli. In fact, this effect was so robust that it allowed a classification of hits and misses based on the single-trial EEG signal (see ROC curves in Fig. 2). Thus, even if subjects were instructed to monitor and report stimuli only at the cued location while completely ignoring the uncued location, the single-trial EEG response could provide a proxy for perception on the unattended side even without a behavioral report. The crucial question would then be whether prestimulus phase is differentially correlated with the poststimulus EEG response for attended and (now completely) unattended stimuli.

Attention-driven periodic sampling is reminiscent of a "serial" visual search mechanism. In serial search, attention is directed to one item at a time, and the attentional spotlight shifts from item (s) to item(s) until the target is found or the search is terminated $(11,12)$. A possible neural basis of serial shifts of attention has been identified in a recent study, which investigated local field potentials in monkeys performing a visual search task (10). It was found that covert shifts of attention (i.e., shifts that do not involve eye movements) were locked to oscillations in the frontal eye fields, such that each cycle of the oscillation corresponded to a distinct item. These oscillations occurred in the $\beta$ frequency range $(18-34 \mathrm{~Hz})$, but the exact frequency was variable across trials and correlated with single-trial reaction times. Thus, the authors suggested that the speed of attentional shifts might be controlled by the frequency of the underlying oscillations (10). One important implication of these results is that attention cannot be allocated at any given time but only at specific phases of the oscillatory cycle. This idea is in line with the present findings, although the difference in frequency bands $(7-10 \mathrm{~Hz}$ in our case, $18-34 \mathrm{~Hz}$ in ref. 10) may require further elucidating.

Another surprising implication of our findings is that attention may operate periodically not only when multiple items are to be searched. A recent study by VanRullen et al. (8) proposed on the basis of psychophysical models that information could be sampled periodically at a rate of approximately seven items per second, even when only a single location has to be monitored (as in the present study). Moreover, illusory motion reversals in the continuous wagon-wheel illusion, which are thought to be caused by a periodic sampling mechanism $(9,13,22)$, are much decreased when the moving stimuli are not attended (9). The present findings that focusing attention at a single location leads to periodic rather than sustained facilitation of perception (and that no such periodic modulation of performance is found at unattended locations) could reflect the physiological manifestations of these psychophysical effects.

Mathewson et al. (24) recently put forward a "pulsed inhibition" account of $\alpha$ oscillations. This account was based on the observation that effects of $\alpha$ phase on perception were strongest in trials with strong $\alpha$ power. The authors suggested that phase effects reflect a periodic suspension (analoguous to a car's antilock braking system) of the inhibition of visual sensitivity, which is caused by strong $\alpha$ oscillations. A crucial implication of this account would be that effects of phase and power on perception pertain to the same oscillatory process. Contrary to this interpretation, we found that effects of phase and power occurred at different frequencies ( $7 \mathrm{~Hz}$ and $12 \mathrm{~Hz}$, respectively) and had a different scalp distribution (fronto-central and posterior, respectively). In addition, whereas phase effects were strongest for attended stimuli, attention actually decreased $\alpha$ power in the relevant hemisphere. Together, these results imply that effects of phase and power are related to different oscillatory mechanisms.

Another link between attention and periodic brain processes is provided by studies in which oscillatory brain responses were entrained by periodic stimulation. Jones et al. (47) presented sequences of distractor tones that were concluded by test tones. The rhythm of the distractor sequence could be regular or variable, and the test tone could be presented in or out of sync with this rhythm. Accuracy in a pitch judgment task was optimal when the rhythm of the distractor sequence was regular and when the test stimulus was presented at rhythmically expected times. The authors concluded that entraining rhythms attract attention in time by inducing temporal expectancies about stimulus onsets (47). Lakatos et al. (19) investigated the neurophysiological mechanisms of attentional entrainment. Monkeys were presented with concurrent rhythmic streams of auditory and visual stimuli. On separate trials, the monkeys had to detect rare targets either in the visual or in the auditory stream. The authors found that neurons in V1 were entrained by the attended stream such that neural responses to attended target stimuli fell into the high-excitability phase of the entrained rhythm, resulting in larger stimulus evoked responses and faster reaction times. On the basis of these results, it has been suggested that sensory systems can entrain to task-relevant temporal structure as a means for attentional selection (32). A similar proposal suggests that internal oscillations or "attending rhythms," which generate expectancies for future events, can be entrained by external stimulus rhythms (48). In the present study, the stimulus sequence had no regular temporal structure and so neural oscillations were not entrained by the external input. However, the present results demonstrate that even in the absence of an external entraining rhythm, internal brain oscillations affect perceptual sensitivity for attended stimuli. This indicates that when stimuli occur unpredictably, the brain does not operate in a completely continuous mode and that sustained attention may have a natural or "default" periodicity around $7 \mathrm{~Hz}$. In other words, the spotlight of sustained attention does not shine continuously-it blinks on and off every 100-150 ms.

\section{Materials and Methods}

Subjects. Seventeen participants volunteered after giving written informed consent. Two participants were excluded from the analysis because of excessive artifacts in the EEG data, which contaminated more than $50 \%$ of the trials. Two other subjects were excluded owing to poor behavioral performance $\left(d^{\prime}<0.5\right)$, which prevented the staircase from converging, leaving 13 subjects in the sample ( 5 female; mean age $26 \mathrm{y} ; 11$ right handed). All par- 
ticipants had normal or corrected-to-normal vision. The experimental protocol was approved by the Centre National de la Recherche Scientifique ethics committee.

Stimuli and Procedure. The experiment was written in Matlab using the Psychophysics Toolbox (49). All stimuli were presented on a black background on a $160-\mathrm{Hz}$ cathode ray tube monitor. Subjects performed a demanding visual detection task. Each run started with the presentation of a central arrow pointing leftward or rightward, instructing participants to focus attention at the indicated location while maintaining central fixation. Targets were then presented either at the cued location (valid; attended) or at the uncued location (invalid; unattended). A target was a very small point of light (diameter: $7^{\prime}$ visual angle) presented for $6 \mathrm{~ms}$ at an eccentricity of $5.7^{\circ}$ visual angle to the left or to the right of the central fixation cross. Each run comprised nine randomly interleaved trials: two for each combination of cue validity and hemifield and one catch trial without actual stimulus presentation. The interstimulus intervals varied randomly from 1,500 to 1,900 ms. Participants were instructed to press the space bar as fast as possible whenever they detected any stimulus, irrespective of the stimulus' location.

The targets' luminance was determined with an adaptive staircase procedure (50), which found the individual luminance threshold at which $50 \%$ of the stimuli were detected. Four separate staircases were run: one for each combination of cue validity and hemifield. Thus, although the hit rate is expected to be identical for attended and unattended targets in both hemifields, effects of attention would manifest as lower luminance thresholds for attended stimuli.

EEG Acquisition. Continuous EEG was acquired with a 64-channel ActiveTwo system (Biosemi). Two additional electrodes [CMS (common mode sense) and DRL (driven right leg)] were used as reference and ground. Electrodes were placed according to the international $10-10$ system. The vertical and horizontal electrooculogram were recorded by attaching additional electrodes above and below the left eye and at the outer canthi of both eyes. An active electrode (CMS) and a passive electrode (DRL) were used to compose a feedback loop for amplifier reference. Details of this circuitry can be found on the Biosem website (www.biosemi.com/faq/cms\&drl.htm). All signals were digitized at $1,024 \mathrm{~Hz}$, 24-bit A/D conversion, and filtered between $100 \mathrm{~Hz}$ and $0.16 \mathrm{~Hz}$. Data were downsampled offline to $512 \mathrm{~Hz}$, converted to an average reference, and epoched from $-1,200 \mathrm{~ms}$ before to $900 \mathrm{~ms}$ after stimulus onset. An automatic artifact rejection excluded epochs in which the signal exceeded $\pm 75 \mu \mathrm{V}$, and the remaining data were screened manually for residual artifacts.

Event-Related Potentials. ERPs were computed as the average across all trials per condition and baseline corrected by subtracting the average of the 400-ms prestimulus baseline. ERPs were tested statistically after data were averaged across channels in a posterior-parietal region of interest (Fig. 2) and sampling points in the time windows from $200 \mathrm{~ms}$ to $270 \mathrm{~ms}$ and from $400 \mathrm{~ms}$ to $520 \mathrm{~ms}$. A repeated-measures ANOVA tested for effects of perception (hits vs. misses) and of attention (valid/attended vs. invalid/unattended).

Time-Frequency Analysis. The analysis focused on effects of perception (hits vs. misses) and attention (attended vs. unattended stimuli) on phase dis tributions and spectral power of the EEG. Phase and power were computed by means of a continuous wavelet transform of single-trial data for the

1. Desimone R, Duncan J (1995) Neural mechanisms of selective visual attention. Annu Rev Neurosci 18:193-222.

2. Reynolds JH, Chelazzi L (2004) Attentional modulation of visual processing. Annu Rev Neurosci 27:611-647.

3. Carrasco M, McElree B (2001) Covert attention accelerates the rate of visual information processing. Proc Natl Acad Sci USA 98:5363-5367.

4. Ling S, Carrasco M (2006) Sustained and transient covert attention enhance the signal via different contrast response functions. Vision Res 46:1210-1220.

5. Carrasco M (2006) Covert attention increases contrast sensitivity: Psychophysical, neurophysiological and neuroimaging studies. Prog Brain Res 154:33-70.

6. Corbetta M, Shulman GL (2002) Control of goal-directed and stimulus-driven attention in the brain. Nat Rev Neurosci 3:201-215.

7. Nakayama K, Mackeben M (1989) Sustained and transient components of focal visual attention. Vision Res 29:1631-1647.

8. VanRullen R, Carlson T, Cavanagh P (2007) The blinking spotlight of attention. Proc Natl Acad Sci USA 104:19204-19209.

9. VanRullen R, Reddy L, Koch C (2005) Attention-driven discrete sampling of motion perception. Proc Natl Acad Sci USA 102:5291-5296.

10. Buschman TJ, Miller EK (2009) Serial, covert shifts of attention during visual search are reflected by the frontal eye fields and correlated with population oscillations. Neuron 63:386-396. frequency range from 2 to $50 \mathrm{~Hz}$. The length of the wavelets increased linearly from two cycles at $2 \mathrm{~Hz}$ to eight cycles at $50 \mathrm{~Hz}$. This modified wavelet transform was selected to optimize the tradeoff between temporal resolution at lower frequencies and stability at higher frequencies. At each time $t$ and frequency $f$, the result of the wavelet transform for trial $k$ is a complex number in which $A$ represents the amplitude of the signal and $\varphi$ its phase:

$$
A_{k(t, f)} e^{i \varphi_{k(t, f)}}
$$

Analysis of Phase. A possible link between prestimulus phase and perception was investigated by exploiting the strong correlation between perception and the ERP. Although large visual evoked ERPs were observed in response to hits, no ERPs were evoked by misses (Fig. 2). If prestimulus phases were in fact predictive of perception, they should thus highly correlate with the amplitude of the poststimulus ERP across single trials. To avoid basing this computation on an arbitrary selection of electrodes, we used the GFP as a summary measure of the ERP across the scalp (51). For each sampling point, the GFP is computed as the SD of the ERP across all electrodes. To find the time range in which the GFP had the strongest association with perception, we conducted a ROC analysis (SI Materials and Methods). We then computed for each time-frequency point at each electrode the circular-linear correlation between phases and the magnitude of the GFP in the time window where the area under the ROC curve was maximal for each given subject (SI Materials and Methods).

The exact impact of prestimulus phase on GFP and perception was further assessed by pooling single trials according to the phase at that timefrequency point and computing each observer's average GFP and detection rate in each of 11 bins (Fig. 4). To minimize the impact of individual variations, GFP and detection rates were standardized for each observer by dividing the GFP and hit rate in each bin by the average of that observer Because the exact phase at which GFP was largest could vary slightly between subjects, we adjusted each subject's phase distribution: phases were shifted such that for each subject, the phase at which GFP was largest (averaged across attended and unattended stimuli) was aligned to a phase angle of zero. The same phase alignment was used in the analysis of phase and perception, allowing us to assess whether the same phase, which yields largest GFP responses, is also predictive for perception. These distributions were computed for each of the channels in a fronto-central region of interest (comprising channels F1, Fz, F2, FC1, FCz, and FC3) and were subsequently averaged. A repeated-measures ANOVA tested for effects of attention (valid/attended vs. invalid/unattended) and phase (10 phase bins). Because of the alignment of phase distributions, the largest GFP and optimal performance is always expected for the central bin. Therefore, this bin was not included in the statistical analysis.

Analysis of Spectral Power. Spectral power was tested for effects of perception by computing two-sample $t$ tests between hits and misses after data were averaged across a time-frequency window from -400 to $0 \mathrm{~ms}$. Likewise, effects of attention were analyzed by a $t$ test between electrodes contralateral and ipsilateral to the attended location (Fig. S5).

ACKNOWLEDGMENTS. This work was supported by Agence Nationale de la Recherche Grant 06JCJC-0154 and a European Young Investigator Award (to R.V.).

11. Treisman AM, Gelade G (1980) A feature-integration theory of attention. Cognit Psychol 12:97-136.

12. Wolfe J (1998) Attention, ed Pashler H (Psychology Press, Hove, UK), pp 13-73.

13. Purves D, Paydarfar JA, Andrews TJ (1996) The wagon wheel illusion in movies and reality. Proc Natl Acad Sci USA 93:3693-3697.

14. VanRullen R, Reddy L, Koch C (2006) The continuous wagon wheel illusion is associated with changes in electroencephalogram power at approximately $13 \mathrm{~Hz}$. J Neurosci 26:502-507.

15. Bishop GH (1932) Cyclic changes in excitability of the optic pathway of the rabbit. Am J Physiol 103:213-224.

16. Buzsáki G, Draguhn A (2004) Neuronal oscillations in cortical networks. Science 304 1926-1929.

17. Lakatos $\mathrm{P}$, et al. (2005) An oscillatory hierarchy controlling neuronal excitability and stimulus processing in the auditory cortex. J Neurophysio/ 94:1904-1911.

18. Fries $P$, Nikolić D, Singer W (2007) The gamma cycle. Trends Neurosci 30:309-316.

19. Lakatos P, Karmos G, Mehta AD, Ulbert I, Schroeder CE (2008) Entrainment of neuronal oscillations as a mechanism of attentional selection. Science 320:110-113.

20. Rajkai C, et al. (2008) Transient cortical excitation at the onset of visual fixation. Cereb Cortex 18:200-209.

21. Sirota A, et al. (2008) Entrainment of neocortical neurons and gamma oscillations by the hippocampal theta rhythm. Neuron 60:683-697. 
22. VanRullen R, Koch C (2003) Is perception discrete or continuous? Trends Cogn Sci 7 207-213.

23. Busch NA, Dubois J, VanRullen R (2009) The phase of ongoing EEG oscillations predicts visual perception. J Neurosci 29:7869-7876.

24. Mathewson KE, Gratton G, Fabiani M, Beck DM, Ro T (2009) To see or not to see: prestimulus alpha phase predicts visual awareness. J Neurosci 29:2725-2732.

25. Fisher NI (1995) Statistical Analysis of Circular Data (Cambridge Univ Press, Cambridge, UK).

26. Zar J (1999) Biostatistical Analysis (Prentice Hall, Upper Saddle River, NJ), 4th Ed.

27. Ergenoglu T, et al. (2004) Alpha rhythm of the EEG modulates visual detection performance in humans. Brain Res Cogn Brain Res 20:376-383.

28. Hanslmayr S, et al. (2007) Prestimulus oscillations predict visual perception performance between and within subjects. Neuroimage 37:1465-1473.

29. van Dijk H, Schoffelen JM, Oostenveld R, Jensen O (2008) Prestimulus oscillatory activity in the alpha band predicts visual discrimination ability. J Neurosci 28 1816-1823.

30. Thut G, Nietzel A, Brandt SA, Pascual-Leone A (2006) Alpha-band electroencephalographic activity over occipital cortex indexes visuospatial attention bias and predicts visual target detection. J Neurosci 26:9494-9502.

31. Worden MS, Foxe JJ, Wang N, Simpson GV (2000) Anticipatory biasing of visuospatial attention indexed by retinotopically specific alpha-band electroencephalography increases over occipital cortex. J Neurosci 20:RC63.

32. Schroeder CE, Lakatos $P$ (2009) Low-frequency neuronal oscillations as instruments of sensory selection. Trends Neurosci 32:9-18.

33. Barry RJ, et al. (2004) Event-related potentials in the auditory oddball as a function of EEG alpha phase at stimulus onset. Clin Neurophysiol 115:2593-2601.

34. Haig AR, Gordon E (1998) EEG alpha phase at stimulus onset significantly affects the amplitude of the P3 ERP component. Int J Neurosci 93:101-115.

35. Jansen BH, Brandt ME (1991) The effect of the phase of prestimulus alpha activity on the averaged visual evoked response. Electroencephalogr Clin Neurophysiol 80 241-250.

36. Kruglikov SY, Schiff SJ (2003) Interplay of electroencephalogram phase and auditory evoked neural activity. J Neurosci 23:10122-10127.
37. Makeig S, et al. (2002) Dynamic brain sources of visual evoked responses. Science 295: 690-694.

38. Callaway E (1960) Yeager CL, 3rd (1960) Relationship between reaction time and electroencephalographic alpha phase. Science 132:1765-1766.

39. Dustman RE, Beck EC (1965) Phase of alpha brain waves, reaction time and visually evoked potentials. Electroencephalogr Clin Neurophysiol 18:433-440.

40. Varela FJ, Toro A, John ER, Schwartz EL (1981) Perceptual framing and cortical alpha rhythm. Neuropsychologia 19:675-686.

41. Stroud JN (1967) The fine structure of psychological time. Ann N Y Acad Sci 138: 623-631.

42. Smith ML, Gosselin F, Schyns PG (2006) Perceptual moments of conscious visual experience inferred from oscillatory brain activity. Proc Natl Acad Sci USA 103: 5626-5631.

43. Cameron EL, Tai JC, Carrasco M (2002) Covert attention affects the psychometric function of contrast sensitivity. Vision Res 42:949-967.

44. Heinze HJ, Luck SJ, Mangun GR, Hillyard SA (1990) Visual event-related potentials index focused attention within bilateral stimulus arrays. I. Evidence for early selection. Electroencephalogr Clin Neurophysiol 75:511-527.

45. Luck SJ, Woodman GF, Vogel EK (2000) Event-related potential studies of attention. Trends Cogn Sci 4:432-440.

46. Klimesch W, Sauseng P, Hanslmayr S (2007) EEG alpha oscillations: The inhibitiontiming hypothesis. Brain Res Brain Res Rev 53:63-88.

47. Jones MR, Moynihan H, MacKenzie N, Puente J (2002) Temporal aspects of stimulusdriven attending in dynamic arrays. Psychol Sci 13:313-319.

48. Large E, Jones MR (1999) The dynamics of attending: How people track time-varying events. Psychol Rev 106:119-159.

49. Brainard DH (1997) The psychophysics toolbox. Spat Vis 10:433-436

50. Watson AB, Pelli DG (1983) QUEST: A Bayesian adaptive psychometric method. Percept Psychophys 33:113-120.

51. Murray MM, Brunet D, Michel CM (2008) Topographic ERP analyses: A step-by-step tutorial review. Brain Topogr 20:249-264. 